\title{
Performance and Robustness Analysis of Sequential Hypotheses Testing for Time Series with Trend
}

\author{
Alexey Kharin \\ Belarusian State Universitiy
}

\author{
Ton That Tu \\ Belarusian State Universitiy, \\ Da Nang University of Education
}

\begin{abstract}
The problem of sequential testing of simple hypotheses for time series with a trend is considered. Analytic expressions and asymptotic expansions for error probabilities and expected numbers of observations are obtained. Robustness analysis is performed. Numerical results are given.
\end{abstract}

Keywords: sequential test, time series with trend, error probabilities, expected sample sizes, asymptotic expansions.

\section{Introduction}

The sequential approach to test parametric hypotheses proposed by Wald (see Wald (1947)) has been applied in many practical problems of computer data analysis. The sequential probability ratio test (SPRT) is proved to be optimal in terms of minimizing expected sample size under the assumption that type I and type II error probabilities do not exceed preassigned values (see Wald and Wolfowitz (1948)). The problem of sequential test performance characteristics (error probabilities and expected number of observations) evaluation is well studied for the case of identical distribution of observations (see Govindarajulu (2004), Kharin (2013), Kharin (2016)). In this paper, the model of non-identical distribution is considered for the problem of two simple hypotheses testing (see Kharin and Ton (2016)).

In practice, data does not often follow the hypothetical model exactly (see Huber (1981), Kharin (2005)), and the problem of robustness under distortions (see Kharin (1997), Maevskii and Kharin (2002)) is important for sequential testing (see Kharin (2011), Kharin and Kishylau (2015)). Here we consider the problem of robustness of sequential tests for time series with trend.

\section{Mathematical model}

Let $x_{1}, x_{2}, \ldots$ be time series with a trend:

$$
x_{t}=\theta^{T} \psi(t)+\xi_{t}, t=1,2,3, \ldots,
$$


where $\psi(t)=\left(\psi_{1}(t), \psi_{2}(t), \ldots, \psi_{m}(t)\right)^{T}, t \geq 1$, are the vectors of basic functions of trend, $\theta=\left(\theta_{1}, \theta_{2}, \ldots, \theta_{m}\right)^{T} \in \mathbb{R}^{m}$ is an unknown vector of coefficients, and $\left\{\xi_{t}, t \geq 1\right\}$ is the sequence of independent identically distributed random variables, $\xi_{t} \sim N\left(0, \sigma^{2}\right)$.

Consider two simple hypotheses $\left(\theta^{0}, \theta^{1} \in \mathbb{R}^{m}\right.$ are known vectors):

$$
H_{0}: \theta=\theta^{0}, H_{1}: \theta=\theta^{1} \text {. }
$$

Denote the accumulated log-likelihood ratio statistic:

$$
\Lambda_{n}=\Lambda_{n}\left(x_{1}, x_{2}, \ldots, x_{n}\right)=\sum_{t=1}^{n} \lambda_{t}
$$

where $\lambda_{t}=\ln \left(\frac{p_{t}\left(x_{t}, \theta^{1}\right)}{p_{t}\left(x_{t}, \theta^{0}\right)}\right)$ is the log-likelihood ratio calculated on observation $x_{t}$, and $p_{t}(x, \theta)$ is the probability density function of $x_{t}$ provided the true parameter value is $\theta$. To test hypotheses (2), after $\mathrm{n}$ observations one makes the decision:

$$
d=\mathbf{1}_{\left[C_{+},+\infty\right)}\left(\Lambda_{n}\right)+2 \cdot \mathbf{1}_{\left(C_{-}, C_{+}\right)}\left(\Lambda_{n}\right) .
$$

Thresholds $C_{-}$and $C_{+}$are the parameters of the test. Decisions $d=0$ and $d=1$ mean stopping of the observation process and acceptance of $H_{0}$ or $H_{1}$ correspondently. According to Wald (1947),

$$
C_{+}=\ln \left(\left(1-\beta_{0}\right) / \alpha_{0}\right), C_{-}=\ln \left(\beta_{0} /\left(1-\alpha_{0}\right)\right),
$$

where $\alpha_{0}, \beta_{0}$ are given values for error probabilities of types I and II respectively.

\section{Some auxiliary results}

Let $\zeta_{n}, n \geq 1$ be a sequence of random variables satisfying the following conditions:

i) $\zeta_{n} \in T=\{0,1,2, \ldots, K, K+1\}, n \in \mathbb{N}$;

ii) $P\left(\zeta_{n}=i_{1} \mid \zeta_{k}=i_{1}\right)=1, i_{1} \in\{0,1\}, n>k$;

iii) $P\left(\zeta_{n}=i_{1} \mid \zeta_{k}=i_{2}, \zeta_{l}=i_{3}\right)=P\left(\zeta_{n}=i_{1} \mid \zeta_{k}=i_{2}\right), n>k>l \geq 1, i_{1}, i_{2}, i_{3} \in T$.

Remark 1. A Markov chain with a finite state space $T$, in which the states 0,1 are absorbing, satisfies conditions (5)-(7).

Introduce the notation:

$$
\begin{gathered}
T_{1}=\{0,1\}, T_{2}=\{2,3, \ldots, K, K+1\}, T=T_{1} \cup T_{2}, \\
P(k)=\left\{p_{i j}(k)\right\}_{(K+2) \times(K+2)}, P(k, l)=\left\{p_{i j}(k, l)\right\}_{(K+2) \times(K+2)}, \\
p_{i j}(k)=P\left(\zeta_{k}=j \mid \zeta_{k-1}=i\right), p_{i j}(k, l)=P\left(\zeta_{k+l}=j \mid \zeta_{k}=i\right) .
\end{gathered}
$$

Since (6), matrices $P(k)$ and $P(1, k)$ can be expressed as follows:

$$
P(k)=\left(\begin{array}{c|c}
I_{2} & O_{2 \times K} \\
\hline R_{k} & Q_{k}
\end{array}\right), k \geq 2 ; \quad P(1, k)=\left(\begin{array}{c|c}
I_{2} & O_{2 \times K} \\
\hline \bar{R}_{k} & \bar{Q}_{k}
\end{array}\right), k \geq 1,
$$

where $R_{k}, \bar{R}_{k}$ are some matrices of size $K \times 2, I_{k}$ is the identity matrix of size $k, O_{2 \times K}$ is the $2 \times K$-matrix with all elements equal to 0 , and $Q_{k}, \bar{Q}_{k}$ are some matrices of size $K \times K$. For $k<n<k+l$ we have:

$$
p_{i j}(k, l)=\sum_{t \in T} P\left(\zeta_{n}=t \mid \zeta_{k}=i\right) P\left(\zeta_{k+l}=j \mid \zeta_{n}=t\right)=\sum_{t \in T} p_{i t}(k, n-k) p_{t j}(n, k+l-n),
$$

which implies that

$$
P(k, l)=P(k, n-k) P(n, k+l-n), k<n<k+l .
$$


Therefore,

$$
P(1, k)=P(2) P(3) \ldots P(k+1), k \geq 1 .
$$

From (8) and (10), we get $\left(Q_{1}=I_{K}\right)$ :

$$
\bar{Q}_{k}=Q_{1} Q_{2} \ldots Q_{k} Q_{k+1} .
$$

Let $t$ be the total number of time moments for which process $\zeta_{n}$ belongs to $T_{2} ; n_{j}\left(j \in T_{2}\right)$ be the number of time moments for which $\zeta_{n}=j ; u_{j}^{k}$ be the function that is 1 if the process $\zeta_{n}=j$ after $k$ steps, and is 0 otherwise; $E_{i}(\cdot)$ be the conditional expected value given $\zeta_{1}=i$. Denote: $N=\sum_{k=0}^{+\infty} \bar{Q}_{k} ; \tau=N \mathbf{1}_{K} ; \mathbf{1}_{K}$ is the vector of size $K$ with all elements equal to 1 .

Theorem 1. In the above notation, for sequence (5)-(7) the following equations are satisfied:

$$
\left\{E_{i}\left(n_{j}\right)\right\}_{K \times K}=N, i, j \in T_{2},\left\{E_{i}(t)\right\}_{K \times 1}=\tau, i \in T_{2} .
$$

Proof. Consider representation $n_{j}=\sum_{k=0}^{+\infty} u_{j}^{k}$. Therefore,

$$
\begin{aligned}
\left\{E_{i}\left(n_{j}\right)\right\} & =\left\{\sum_{k=0}^{+\infty} E_{i}\left(u_{j}^{k}\right)\right\}=\sum_{k=0}^{+\infty}\left\{p_{i j}(1, k)\right\}=\sum_{k=0}^{+\infty} \bar{Q}_{k} \\
\left\{E_{i}(t)\right\} & =\left\{\sum_{j \in T_{2}} E_{i}\left(n_{j}\right)\right\}=N \mathbf{1}_{K}=\tau .
\end{aligned}
$$

Let $B$ be a matrix of size $K \times 2, B=\left\{b_{i j}\right\}_{K \times 2}$, where $b_{i j}$ is the probability that the sequence $\zeta_{n}$ started in $i$ is absorbed in $j, i \in T_{2}, j \in T_{1}$.

Theorem 2. If conditions (5)-(7) hold for $\zeta_{n}$, then

$$
B=\sum_{k=1}^{+\infty} \bar{Q}_{k-1} R_{k+1} .
$$

Proof. Let $B(k), k \geq 1$, be matrix of size $K \times 2, B(k)=\left\{b_{i j}(k)\right\}_{K \times 2}$, where $b_{i j}(k)$ is the probability that the process $\zeta_{n}$ starting in $i$ is absorbed in $j$ after exactly $k$ steps, $i \in T_{2}, j \in T_{1}$. We obtain (13) from the facts that $B(k)=\bar{Q}_{k-1} R_{k+1}$ and $B=\sum_{k=1}^{+\infty} B(k)$.

Corollary 1. If $\pi=\left(\pi_{0}, \pi_{1}, \ldots, \pi_{K}, \pi_{K+1}\right), \pi_{i}=P\left(\zeta_{1}=i\right), i \in T$ and $\pi^{\prime}=\left(\pi_{2}, \ldots, \pi_{K}, \pi_{K+1}\right)$, then the total expected value $E(t)$ equals:

$$
E(t)=\pi^{\prime} \tau
$$

Let $U, V, T \in \mathbb{R}$ be three independent random variables, $U \sim N\left(\mu_{u}, \sigma_{u}^{2}\right), V \sim N\left(\mu_{v}, \sigma_{v}^{2}\right), T \sim$ $N\left(\mu_{t}, \sigma_{t}^{2}\right)$. From the properties of multivariate normal distributions (see Bilodeau and Brenner (1999)), we have:

$$
\begin{aligned}
& f_{U+V \mid V}(x \mid y)=\frac{f_{U+V, V}(x, y)}{f_{V}(y)}=n_{1}\left(x ; y+\mu_{u}, \sigma_{u}^{2}\right), \\
& f_{U+V+T \mid V+T, T}(x \mid y, z)=f_{U+V+T \mid V+T}(x \mid y)=n_{1}\left(x ; y+\mu_{u}, \sigma_{u}^{2}\right), \\
& \left|f_{U}^{\prime}(x)\right| \leq \frac{e^{-1 / 2}}{\sigma_{u}^{2} \sqrt{2 \pi}}, \quad \forall x \\
& \left|f_{U+V \mid V}^{\prime}(x \mid y)\right| \leq \frac{e^{-1 / 2}}{\sigma_{u}^{2} \sqrt{2 \pi}}, \quad \forall x, y .
\end{aligned}
$$


Lemma 1. (Gut 2005) If $X$ is a non-negative, integer valued random variable, then

$$
E(X)=\sum_{n=1}^{+\infty} P(X \geq n) .
$$

Lemma 2. (Gut 2005) Let $r>0$, and suppose that $X$ is a non-negative random variable. Then the following inequalities hold:

$$
\sum_{n=1}^{+\infty} n^{r-1} P(X \geq n) \leq E\left(X^{r}\right) \leq 1+\sum_{n=1}^{+\infty} n^{r-1} P(X \geq n),
$$

and

$$
E\left(|X|^{r}\right)<\infty \text { if and only if } \sum_{n=1}^{+\infty} n^{r-1} P(X \geq n)<\infty .
$$

Lemma 3. (Coope 1996) For positive semidefinite matrices $A, B$ of the same order

$$
0 \leq \operatorname{tr}(A B) \leq \operatorname{tr}(A) \operatorname{tr}(B) .
$$

\section{Performance and robustness analysis}

\subsection{Performance analysis for the hypothetical model}

For model (1), (3) we have $(t \geq 1)$ :

$$
\begin{gathered}
x_{t} \sim N\left(\theta^{T} \psi(t) ; \sigma^{2}\right), p_{t}(x, \theta)=\frac{1}{\sigma \sqrt{2 \pi}} \exp \left\{-\frac{1}{2 \sigma^{2}}\left(x-\theta^{T} \psi(t)\right)^{2}\right\} ; \\
\lambda_{t}=\lambda_{t}\left(x_{t}\right)=-\frac{1}{2 \sigma^{2}}\left\{2 x_{t}\left(\theta^{0}-\theta^{1}\right)^{T} \psi(t)+\left(\theta^{1}\right)^{T} \psi(t) \psi^{T}(t) \theta^{1}-\left(\theta^{0}\right)^{T} \psi(t) \psi^{T}(t) \theta^{0}\right\} .
\end{gathered}
$$

Due to the properties of the normal distribution, $\lambda_{t}$ and $\Lambda_{n}$ have also normal distributions with the following parameters:

$$
\begin{gathered}
E\left(\lambda_{t}\right)=-\frac{1}{2 \sigma^{2}}\left\{2\left(\theta^{0}-\theta^{1}\right)^{T} \psi(t) \psi^{T}(t) \theta+\left(\theta^{1}\right)^{T} \psi(t) \psi^{T}(t) \theta^{1}-\left(\theta^{0}\right)^{T} \psi(t) \psi^{T}(t) \theta^{0}\right\}, \\
D\left(\lambda_{t}\right)=\frac{\left(\theta^{0}-\theta^{1}\right)^{T} \psi(t) \psi^{T}(t)\left(\theta^{0}-\theta^{1}\right)}{\sigma^{2}} \\
E\left(\Lambda_{n}\right)=-\frac{1}{2 \sigma^{2}}\left\{2\left(\theta^{0}-\theta^{1}\right)^{T} H_{n} \theta+\left(\theta^{1}\right)^{T} H_{n} \theta^{1}-\left(\theta^{0}\right)^{T} H_{n} \theta^{0}\right\} \\
D\left(\Lambda_{n}\right)=\frac{\left(\theta^{0}-\theta^{1}\right)^{T} H_{n}\left(\theta^{0}-\theta^{1}\right)}{\sigma^{2}}
\end{gathered}
$$

where $H_{n}=\sum_{t=1}^{n} \psi(t) \psi^{T}(t)$.

Introduce the notation: $E^{(k)}(\cdot), D^{(k)}(\cdot)$ are conditional expected value and variance provided the hypothesis $H_{k}$ is true $(k=0,1), \Phi(\cdot)$ is the cumulative distribution function of the standard normal law,

$$
\begin{aligned}
& \sigma_{n}^{2}=D^{(0)}\left(\lambda_{n}\right)=D^{(1)}\left(\lambda_{n}\right)=\frac{\left(\theta^{0}-\theta^{1}\right)^{T} \psi(n) \psi^{T}(n)\left(\theta^{0}-\theta^{1}\right)}{\sigma^{2}} \\
& \mu_{n}^{(k)}=E^{(k)}\left(\lambda_{n}\right)=\frac{(-1)^{k+1} \sigma_{n}^{2}}{2}, \quad s_{n}^{2}=\sum_{t=1}^{n} \sigma_{t}^{2}, \quad m_{n}^{(k)}=\sum_{t=1}^{n} \mu_{t}^{(k)}=\frac{(-1)^{k+1} s_{n}^{2}}{2} ; \\
& A_{n}=\left\{a_{i j}\right\}_{n \times n}, \quad a_{i j}= \begin{cases}1, & i \geq j, \\
0, & \text { otherwise },\end{cases} \\
& X_{n}=\left(\lambda_{1}, \lambda_{2}, \ldots, \lambda_{n}\right)^{T}, \quad T_{n}=\left(\Lambda_{1}, \Lambda_{2}, \ldots, \Lambda_{n}\right)^{T}=A_{n} X_{n} ; \\
& \mu_{T_{n}}^{(k)}=E^{(k)}\left(T_{n}\right)=A_{n} E^{(k)}\left(X_{n}\right), \quad \Sigma_{T_{n}}=\operatorname{Cov}\left(T_{n}, T_{n}\right)=A_{n} \operatorname{Cov}\left(X_{n}, X_{n}\right) A_{n}^{T} ; \\
& N=\inf \left\{n \in \mathbb{N}: \Lambda_{n} \notin\left(C_{-}, C_{+}\right)\right\}, \quad \Gamma=\left(\theta^{0}-\theta^{1}\right)\left(\theta^{0}-\theta^{1}\right)^{T} .
\end{aligned}
$$


Theorem 3. If $\operatorname{tr}\left(\Gamma H_{n}\right) \rightarrow+\infty$ as $n \rightarrow+\infty$, then test (3)-(4) terminates finitely with probability 1.

Proof. We have:

$$
\begin{aligned}
s_{n}^{2} & =\sum_{t=1}^{n} \sigma_{t}^{2}=\frac{1}{\sigma^{2}} \sum_{t=1}^{n}\left(\theta^{0}-\theta^{1}\right)^{T} \psi(t) \psi^{T}(t)\left(\theta^{0}-\theta^{1}\right) \\
& =\frac{1}{\sigma^{2}} \sum_{t=1}^{n} \operatorname{tr}\left\{\left(\theta^{0}-\theta^{1}\right)^{T} \psi(t) \psi^{T}(t)\left(\theta^{0}-\theta^{1}\right)\right\}=\frac{1}{\sigma^{2}} \operatorname{tr}\left(\Gamma H_{n}\right) .
\end{aligned}
$$

Under the theorem condition, we get $s_{n}^{2} \rightarrow+\infty$ as $n \rightarrow+\infty$. Furthermore, we also have $P_{k}(N>n)=P_{k}\left(\Lambda_{i} \in\left(C_{-}, C_{+}\right), i=\overline{1, n}\right), k=0,1$, and

$$
\begin{aligned}
P_{k}\left(\Lambda_{i} \in\left(C_{-}, C_{+}\right), i=\overline{1, n}\right) & \leq P_{k}\left(\Lambda_{n} \in\left(C_{-}, C_{+}\right)\right)= \\
& =\Phi\left(\frac{C_{+}-\mu_{n}^{(k)}}{s_{n}}\right)-\Phi\left(\frac{C_{-}-\mu_{n}^{(k)}}{s_{n}}\right) \\
& =\Phi\left(\frac{2 C_{+}-(-1)^{k+1} s_{n}^{2}}{2 s_{n}}\right)-\Phi\left(\frac{2 C_{-}-(-1)^{k+1} s_{n}^{2}}{2 s_{n}}\right),
\end{aligned}
$$

which implies that $\lim _{n \rightarrow+\infty} P_{k}\left(\Lambda_{i} \in\left(C_{-}, C_{+}\right), i=\overline{1, n}\right)=0$ or $\lim _{n \rightarrow+\infty} P_{k}(N>n)=0$.

Therefore, $P_{k}(N<+\infty)=1-P_{k}(N=+\infty)=1-\lim _{n \rightarrow+\infty} P_{k}(N>n)=1$.

Corollary 2. Under the conditions of Theorem 3 we get:

$$
\sum_{i=1}^{n} \sum_{j=1}^{m} \psi_{j}^{2}(i) \rightarrow+\infty \text { as } n \rightarrow+\infty .
$$

Additionally, if $\left(\theta_{i}^{1}-\theta_{i}^{0}\right)^{T} \psi_{i}(t), i=\overline{1, m}$, are simultaneously nonnegative (or nonpositive) functions at $t$, then the result of Theorem 3 still holds if $\sum_{i=1}^{n} \psi_{k}^{2}(i) \rightarrow+\infty$, where $k$ is such an index that $\theta_{k}^{1} \neq \theta_{k}^{0}$.

Proof. Note that $\Gamma$ and $H_{n}$ are positive semi-definite matrices. The proof is derived directly from Lemma 3 and the facts that:

$$
\operatorname{tr}\left(H_{n}\right)=\sum_{i=1}^{n} \sum_{j=1}^{m} \psi_{j}^{2}(i), \operatorname{tr}\left(\Gamma H_{n}\right)=\sum_{i=1}^{n}\left(\sum_{k=1}^{m}\left(\theta_{k}^{0}-\theta_{k}^{1}\right) \psi_{k}(i)\right)^{2} .
$$

Remark 2. If $\sum_{i=1}^{n} \sum_{j=1}^{m} \psi_{j}^{2}(i)$ is bounded, then there exists a positive constant $L$ such that $s_{n}^{2} \rightarrow L$ as $n \rightarrow+\infty$. In this case, we get $\sigma_{n} \rightarrow 0$ and $\mu_{n}^{(k)} \rightarrow 0, k=0,1$. It means $\lambda_{n} \stackrel{P}{\rightarrow} 0$ as $n \rightarrow+\infty$ under hypothesis $H_{0}$ or $H_{1}$. In addition, we also have:

$$
\lim _{n \rightarrow+\infty} P_{k}\left(\Lambda_{n} \in\left(C_{-}, C_{+}\right)\right)=\Phi\left(\frac{2 C_{+}-(-1)^{k+1} L}{2 \sqrt{L}}\right)-\Phi\left(\frac{2 C_{-}-(-1)^{k+1} L}{2 \sqrt{L}}\right)>0 .
$$

Theorem 4. Under the conditions of Theorem 3 the following expressions are valid for the characteristics of test (3), (4):

$$
\begin{gathered}
E^{(k)}(N)=1+\sum_{i=1}^{+\infty} \int_{C_{-}}^{C_{+}} d s_{i} \int_{C_{-}}^{C_{+}} d s_{i-1} \ldots \int_{C_{-}}^{C_{+}} n_{i}\left(s ; \mu_{T_{i}}^{(i)}, \Sigma_{T_{i}}\right) d s_{1}, k=0,1, \\
\alpha=\int_{C_{+}}^{+\infty} n_{1}\left(s_{1} ; \mu_{1}^{(0)}, \sigma_{1}^{2}\right) d s_{1}+\sum_{i=2}^{+\infty} \int_{C_{+}}^{+\infty} d s_{i} \int_{C_{-}}^{C_{+}} d s_{i-1} \ldots \int_{C_{-}}^{C_{+}} n_{i}\left(s ; \mu_{T_{i}}^{(0)}, \Sigma_{T_{i}}\right) d s_{1}, \\
\beta=\int_{-\infty}^{C_{-}} n_{1}\left(s_{1} ; \mu_{1}^{(1)}, \sigma_{1}^{2}\right) d s_{1}+\sum_{i=2}^{+\infty} \int_{-\infty}^{C_{-}} d s_{i} \int_{C_{-}}^{C_{+}} d s_{i-1} \ldots \int_{C_{-}}^{C_{+}} n_{i}\left(s ; \mu_{T_{i}}^{(1)}, \Sigma_{T_{i}}\right) d s_{1} .
\end{gathered}
$$


Proof. Under the condition of Theorem 4 the test terminates finitely with probability 1 . From Lemma 1, we have:

$$
E^{(k)}(N)=1+\sum_{i=1}^{+\infty} P_{k}(N>i)=1+\sum_{i=1}^{+\infty} \int_{C_{-}}^{C_{+}} d s_{i} \int_{C_{-}}^{C_{+}} d s_{i-1} \ldots \int_{C_{-}}^{C_{+}} n_{i}\left(s ; \mu_{T_{i}}^{(k)}, \Sigma_{T_{i}}\right) d s_{1} .
$$

For the error type I probability we get:

$$
\begin{aligned}
\alpha & =P_{0}\left(\Lambda_{N} \geq C_{+}\right)=\sum_{i=1}^{+\infty} P_{0}\left(N=i, \Lambda_{N} \geq C_{+}\right) \\
& =P_{0}\left(\Lambda_{1} \geq C_{+}\right)+\sum_{i=2}^{+\infty} P_{0}\left(\Lambda_{i} \geq C_{+}, \Lambda_{j} \in\left(C_{-}, C_{+}\right), j=\overline{1, i-1}\right) .
\end{aligned}
$$

From (23) we get (21). The expression of $\beta$ in (22) is proved analogously.

In practice, it is difficult to use formulae (20)-(22) for computing the characteristics of the test: using numerical methods for approximating the multiple integration in the right hand sides of these equalities is unfeasible. To get upper bounds for these test characteristics, we can use the following estimate:

$$
P\left(\Lambda_{1} \in\left(a_{1}, b_{1}\right), \ldots, \Lambda_{n} \in\left(a_{n}, b_{n}\right)\right) \leq P\left(\Lambda_{i} \in\left(a_{i}, b_{i}\right), \ldots, \Lambda_{n} \in\left(a_{n}, b_{n}\right)\right),
$$

where $i$ is a fixed value in $\{1,2, \ldots, n\}$.

It is obvious that the smaller value $i$, the stricter the inequality (24). In particular, when $\operatorname{tr}\left(\Gamma H_{n}\right)$ tends to $+\infty$ slowly, we should select the value $i$ smaller to get better estimates.

Corollary 3. Under the Theorem 4 condition, the following inequalities hold:

$$
\begin{aligned}
& E^{(k)}(N) \leq\left(\frac{C_{+}-\mu_{1}^{(k)}}{\sigma_{1}}\right)+\Phi\left(\frac{\mu_{1}^{(k)}-C_{-}}{\sigma_{1}}\right) \\
&+\sum_{i=2}^{+\infty} \int_{C_{-}}^{C_{+}} \int_{C_{-}}^{C_{+}} n_{1}\left(x ; m_{i-1}^{(k)}, s_{i-1}^{2}\right) n_{1}\left(y ; x+\mu_{i}^{(k)}, \sigma_{i}^{2}\right) d x d y, k \in\{0,1\}, \\
& \alpha \leq 1-\Phi\left(\frac{C_{+}-\mu_{1}^{(0)}}{\sigma_{1}}\right)+\sum_{i=2}^{+\infty} \int_{C_{+}}^{+\infty} \int_{C_{-}}^{C_{+}} n_{1}\left(x ; m_{i-1}^{(0)}, s_{i-1}^{2}\right) n_{1}\left(y ; x+\mu_{i}^{(0)}, \sigma_{i}^{2}\right) d x d y, \\
& \beta \leq \Phi\left(\frac{C_{-}-\mu_{1}^{(1)}}{\sigma_{1}}\right)+\sum_{i=2}^{+\infty} \int_{-\infty}^{C_{-}} \int_{C_{-}}^{C_{+}} n_{1}\left(x ; m_{i-1}^{(1)}, s_{i-1}^{2}\right) n_{1}\left(y ; x+\mu_{i}^{(1)}, \sigma_{i}^{2}\right) d x d y .
\end{aligned}
$$

Proof. The inequalities are resulting from (24), (15) with $i=n-1$.

Time series data is usually collected at certain intervals. Sometimes, there are patterns that can repeat over fixed periods of time within the data set. Such patterns are known as periodic fluctuations or seasonality. In this case, the function of trend $g(t)=\theta^{T} \psi(t)$ will be periodic with some period $T>0$. In particular, function $h(t)=\left(\theta^{0}-\theta^{1}\right)^{T} \psi(t)$ will also be periodic.

Theorem 5. If there exists an integer $T \geq 1$ such that $h(t+T)=h(t), \forall t=1,2, \ldots$, then the stopping time $N$ has finite moments of any order.

Proof. Without loss of generality, assume that hypothesis $H_{0}$ is true. Due to the Theorem conditions, $\lambda_{t}$ and $\lambda_{t+k T}$ have the same distribution for all $t, k \in \mathbb{N}$.

On event $\{N>k T\}, k \geq 1, \Lambda_{k T}=\lambda_{1}+\ldots+\lambda_{k T} \in\left(C_{-}, C_{+}\right)$, which implies that $\Lambda_{k T}^{2} \leq$ $\left(C_{+}-C_{-}\right)^{2}$. Since $D\left(\Lambda_{k T}\right)=\sum_{i=1}^{k T} D\left(\lambda_{i}\right)=k D\left(\Lambda_{T}\right)=\frac{k}{\sigma^{2}} \operatorname{tr}\left(\Gamma H_{T}\right) \rightarrow+\infty$ as $k \rightarrow+\infty$, there exists $k_{0} \geq 1$ such that on $\left\{N>k_{0} T\right\}$ :

$$
P_{0}\left(\Lambda_{k_{0} T}^{2}<\left(C_{+}-C_{-}\right)^{2}\right)=q \in(0,1) .
$$


Put $n_{0}=h k_{0} T, h \geq 1$. Note that from the fact $N>n_{0}$, we have

$$
\left|\Lambda_{r k_{0} T}-\Lambda_{(r-1) k_{0} T}\right|<C_{+}-C_{-}, \forall r=\overline{1, h} .
$$

Because $\left\{\Lambda_{r k_{0} T}-\Lambda_{(r-1) k_{0} T}, r=\overline{1, h}\right\}$ is a sequence of independent identically distributed random variables and $\left\{N>n_{0}\right\} \subset\left\{\left|\Lambda_{r k_{0} T}-\Lambda_{(r-1) k_{0} T}\right|<C_{+}-C_{-}, \forall r=\overline{1, h}\right\}$, we have

$$
P_{0}\left(N>n_{0}\right) \leq P_{0}\left(\left|\Lambda_{r k_{0} T}-\Lambda_{(r-1) k_{0} T}\right|<C_{+}-C_{-}, \forall r=\overline{1, h}\right)=q^{h}=q^{\frac{n_{0}}{k_{0} T}} .
$$

Therefore, for any $n>k_{0} T$

$$
P_{0}(N>n) \leq q^{\left[\frac{n}{k_{0} T}\right]} \leq q^{\frac{n}{k_{0} T}-1},
$$

which implies $P_{0}(N>n)=o\left(n^{-r}\right)$ as $n \rightarrow+\infty$ for any finite $r$.

The rest part of proof is derived from Lemma 2.

Corollary 4. If the basic vector function of trend $\psi(t)$ is periodic on the set $\mathbb{N}$, then the stopping time $N$ has finite moments of any order.

\subsection{Special case}

Assume that there exists a constant $a \neq 0$ such that $h(t)=a, \forall t \geq 1$, and $H_{0}$ is the true hypothesis.

In this case, $\left\{\lambda_{t}, t \geq 1\right\}$ becomes the sequence of independent and identically distributed random variables from $N\left(\mu, \sigma_{0}^{2}\right)$, where

$$
\mu=\frac{-a^{2}}{2 \sigma^{2}}, \quad \sigma_{0}^{2}=\frac{a^{2}}{\sigma^{2}} .
$$

Let $x$ be a fixed value and put $\Lambda_{n}^{x}=x+\Lambda_{n}$. The new test based on $\Lambda_{n}^{x}$ is equivalent to the original SPRT whose region of indifference is the interval $\left(C_{-}-x, C_{+}-x\right)$. Let $\beta_{\theta}(x)$ and $N_{\theta}(x)$ be the operating characteristic and average sample size functions of this SPRT respectively. From the Markov property of log-likelihood ratio statistic $\Lambda_{n}$ and when $H_{0}$ is true, $\beta_{\theta^{0}}(x)$ and $N_{\theta^{0}}(x)$ are known to satisfy the Fredholm integral equations (see Basseville and Nikiforov (1993), Cox and Miller (1965)):

$$
\begin{gathered}
\beta_{\theta^{0}}(x)=F_{\theta^{0}}\left(C_{-}-x\right)+\int_{C_{-}}^{C_{+}} K_{\theta^{0}}(x, y) \beta_{\theta^{0}}(y) d y, \\
N_{\theta^{0}}(x)=1+\int_{C_{-}}^{C_{+}} K_{\theta^{0}}(x, y) N_{\theta^{0}}(y) d y
\end{gathered}
$$

where $F_{\theta^{0}}(x)=P_{\theta_{0}}\left(\lambda_{1}<x\right)=\Phi\left(\frac{x-\mu}{\sigma_{0}}\right)$ and $K_{\theta^{0}}(x, y)=\frac{\partial}{\partial y} F_{\theta^{0}}(y-x)=\frac{1}{\sigma_{0}} \varphi\left(\frac{y-x-\mu}{\sigma_{0}}\right) ; \varphi(z)=$ $n_{1}(z ; 0,1), z \in \mathbb{R}$.

A numerical method is used for solving these equations. Let $m_{0}$ be a positive integer and $\left\{y_{i}, i=\overline{1, m_{0}}\right\}, C_{-}=y_{1}<y_{2}<\ldots<y_{m_{0}}=C_{+}$, be a partition of the interval $\left[C_{-}, C_{+}\right]$, in which $y_{i}$ having the smallest absolute value is set to be 0 . Note that $\alpha=1-\beta_{\theta^{0}}(0)$ and $E\left(N \mid H_{0}\right)=N_{\theta^{0}}(0)$. Let $\tilde{\beta}_{\theta^{0}}\left(y_{i}\right)$ be approximations of $\beta_{\theta^{0}}(y)$ at $y=y_{i}, i=\overline{1, m_{0}}$. Using the trapezoid formula (see Hoffman (2001)) we have:

$$
\begin{aligned}
\int_{C_{-}}^{C_{+}} K_{\theta^{0}}(x, y) \beta_{\theta^{0}}(y) d y \approx & \sum_{i=1}^{m_{0}-1} \frac{y_{i+1}-y_{i}}{2 \sigma_{0}}\left(\varphi\left(\frac{y_{i}-x-\mu}{\sigma_{0}}\right) \tilde{\beta}_{\theta^{0}}\left(y_{i}\right)+\right. \\
& \left.+\varphi\left(\frac{y_{i+1}-x-\mu}{\sigma_{0}}\right) \tilde{\beta}_{\theta^{0}}\left(y_{i+1}\right)\right) \\
& \approx \sum_{i=1}^{m_{0}} \rho_{i} \varphi\left(\frac{y_{i}-x-\mu}{\sigma_{0}}\right) \tilde{\beta}_{\theta^{0}}\left(y_{i}\right)
\end{aligned}
$$


where

$$
\rho_{i}= \begin{cases}\frac{y_{2}-y_{1}}{2 \sigma_{0}}, & i=1 \\ \frac{y_{m_{0}}-y_{m_{0}-1}}{2 \sigma_{0}}, & i=m_{0} \\ \frac{y_{i+1}-y_{i-1}}{2 \sigma_{0}}, & i \in\left\{2,3, \ldots, m_{0}-1\right\}\end{cases}
$$

In the case of uniform partition, the error of this approximation is $O\left(h^{2}\right)$, where $h=y_{i+1}-y_{i}$ (see Hoffman (2001)). From the approximation above we get the following system of linear equations:

$$
\left\{\begin{array}{clc}
\tilde{\beta}_{\theta^{0}}\left(y_{1}\right) & =\Phi\left(\frac{C_{-}-y_{1}-\mu}{\sigma_{0}}\right)+\sum_{i=1}^{m} \rho_{i} \varphi\left(\frac{y_{i}-y_{1}-\mu}{\sigma_{0}}\right) \tilde{\beta}_{\theta^{0}}\left(y_{i}\right), \\
\vdots & \vdots & \vdots \\
\tilde{\beta}_{\theta^{0}}\left(y_{m_{0}}\right) & =\Phi\left(\frac{C_{-}-y_{m_{0}}-\mu}{\sigma_{0}}\right)+\sum_{i=1}^{m_{0}} \rho_{i} \varphi\left(\frac{y_{i}-y_{m_{0}}-\mu}{\sigma_{0}}\right) \tilde{\beta}_{\theta^{0}}\left(y_{i}\right) .
\end{array}\right.
$$

This system can be represented in the matrix form:

$$
\left(I_{m_{0}}-A\right) \cdot \tilde{\beta}_{\theta^{0}}=B
$$

where

$$
\begin{aligned}
& A=\left\{a_{i j}\right\}_{m_{0} \times m_{0}}, a_{i j}=\rho_{j} \varphi\left(\frac{y_{j}-y_{i}-\mu}{\sigma_{0}}\right), \\
& \tilde{\beta}_{\theta^{0}}=\left(\tilde{\beta}_{\theta^{0}}\left(y_{1}\right), \ldots, \tilde{\beta}_{\theta^{0}}\left(y_{m_{0}}\right)\right)^{T}, \\
& B=\left(\Phi\left(\frac{C_{-}-y_{1}-\mu}{\sigma_{0}}\right), \ldots, \Phi\left(\frac{C_{-}-y_{m_{0}}-\mu}{\sigma_{0}}\right)\right) .
\end{aligned}
$$

Similarly, we also have the system of linear equations for the approximations $\tilde{N}_{\theta^{0}}\left(y_{i}\right)$ of $N_{\theta^{0}}(y)$ at $y=y_{i}, i=\overline{1, m_{0}}$ :

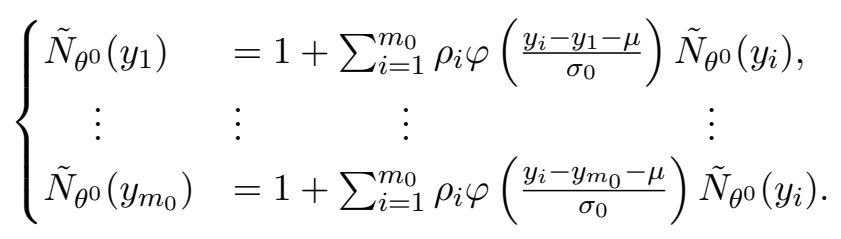

This system is also represented in the matrix form:

$$
\left(I_{m_{0}}-A\right) \cdot \tilde{N}_{\theta^{0}}=\bar{B},
$$

where $\tilde{N}_{\theta^{0}}=\left(\tilde{N}_{\theta^{0}}\left(y_{1}\right), \ldots, \tilde{N}_{\theta^{0}}\left(y_{m_{0}}\right)\right)^{T}$ and $\bar{B}$ is a column vector of size $m_{0}$ whose all elements are equal to one.

\subsection{Approximation of the random sequence $\Lambda_{n}$}

Let us split the state space of $\Lambda_{n}$ on $K+2$ cells:

$$
\begin{gathered}
A_{0}=\left(-\infty, C_{-}\right), A_{i}=\left[C_{i-1}, C_{i}\right), i=\overline{1, K}, A_{K+1}=\left[C_{+},+\infty\right), \\
C_{-}=C_{0}<C_{1}<C_{2}<\ldots<C_{K}=C_{+}, C_{i}=C_{-}+i h, h=\frac{C_{+}-C_{-}}{K}, i=\overline{1, K} .
\end{gathered}
$$

Denote $S_{K}=\left\{A_{1}, A_{2}, \ldots, A_{K}\right\}$.

Lemma 4. If $\inf _{n} \operatorname{tr}\left(\Gamma \psi(n) \psi^{T}(n)\right) \geq C, C=$ const $>0$, then for all $i, j, k, i<j<k$ the following asymptotic expansions are valid:

$$
P\left(\Lambda_{k} \in A^{1} \mid \Lambda_{j} \in A^{2}, \Lambda_{i} \in A^{3}\right)=P\left(\Lambda_{k} \in A^{1} \mid \Lambda_{j} \in A^{2}\right)+R(h),
$$

where $A^{1}, A^{2}, A^{3} \in\left\{A_{0}, A_{1}, \ldots, A_{K}, A_{K+1}\right\}$, and $R(h)= \begin{cases}O(h), & A^{1} \in\left\{A_{0}, A_{K+1}\right\}, \\ O\left(h^{2}\right), & A^{1} \in S_{K} .\end{cases}$ 
Proof. From the Lemma condition and (17)-(18), there exist two positive constants $C_{1}, C_{2}$ such that:

$$
\left|f_{\Lambda_{n}}^{\prime}(x)\right| \leq C_{1}, \forall n \geq 1,\left|f_{\Lambda_{n} \mid \Lambda_{k}}^{\prime}(x \mid y)\right| \leq C_{2}, \forall k<n, \forall x, y \in \mathbb{R} .
$$

The rest part of proof is similar to the proof of Theorem 1 in Kharin (2008).

Denote $f_{C_{-}}^{C_{+}}(x)=\left(\left[\frac{x-C_{-}}{h}\right]+1\right) \cdot \mathbf{1}_{\left(C_{-}, C_{+}\right)}(x)+(K+1) \cdot \mathbf{1}_{\left[C_{+},+\infty\right)}(x)$.

For the random sequence $\Lambda_{n}$ introduce the discrete random sequence $Z_{n}$ with the finite state space $V=\{0,1, \ldots, K+1\}$. Put $Z_{1}=f_{C_{-}}^{C_{+}}\left(\Lambda_{1}\right)$. For $n \geq 2$ :

$$
Z_{n}= \begin{cases}0, & \text { if } Z_{n-1}=0, \\ K+1, & \text { if } Z_{n-1}=K+1, \\ f_{C_{-}}^{C_{+}}\left(\Lambda_{n}\right), & \text { otherwise. }\end{cases}
$$

To simplify the notation, let us renumerate the states space of $Z_{n}$ :

$$
V=\{\{0\},\{K+1\},\{1\}, \ldots,\{K\}\} .
$$

Denote:

$$
\begin{gathered}
P^{(n)}\left(\theta^{k}\right)=\left(\begin{array}{c|c}
I_{2} & \mathbf{O}_{2 \times K} \\
\hline R_{n}\left(\theta^{k}\right) & Q_{n}\left(\theta^{k}\right)
\end{array}\right), k \in\{0,1\}, \\
Q_{n}\left(\theta^{k}\right)=\left\{q_{i j}^{(n)}\left(\theta^{k}\right)\right\}_{K \times K}, R_{n}\left(\theta^{k}\right)=\left\{r_{i j}^{(n)}\left(\theta^{k}\right)\right\}_{K \times 2}, \\
q_{i j}^{(n)}\left(\theta^{k}\right)=\frac{\int_{A_{i}} n_{1}\left(y ; m_{n-1}^{(i)}, s_{n-1}^{2}\right) \int_{A_{j}} n_{1}\left(x ; y+\mu_{n}^{(k)}, \sigma_{n}^{2}\right) d x d y}{\int_{A_{i}} n_{1}\left(y ; m_{n-1}^{(k)}, s_{n-1}^{2}\right) d y}, 1 \leq i, j \leq K, \\
r_{i 1}^{(n)}\left(\theta^{k}\right)=\frac{\int_{A_{i}} n_{1}\left(y ; m_{n-1}^{(k)}, s_{n-1}^{2}\right) \int_{A_{0}} n_{1}\left(x ; y+\mu_{n}^{(k)}, \sigma_{n}^{2}\right) d x d y}{\int_{A_{i}} n_{1}\left(y ; m_{n-1}^{(k)}, s_{n-1}^{2}\right) d y}, 1 \leq i \leq K, \\
r_{i 2}^{(n)}\left(\theta^{k}\right)=\frac{\int_{A_{i}} n_{1}\left(y ; m_{n-1}^{(k)}, s_{n-1}^{2}\right) \int_{A_{K+1}} n_{1}\left(x ; y+\mu_{n}^{(k)}, \sigma_{n}^{2}\right) d x d y}{\int_{A_{i}} n_{1}\left(y ; m_{n-1}^{(k)}, s_{n-1}^{2}\right) d y}, 1 \leq i \leq K, \\
S\left(\theta^{k}\right)=I_{K}+\sum_{i=1}^{+\infty} \prod_{j=1}^{i+1} Q_{j}\left(\theta^{k}\right), \quad B\left(\theta^{k}\right)=R_{2}\left(\theta^{k}\right)+\sum_{i=2}^{+\infty} \prod_{j=1}^{i} Q_{j}\left(\theta^{k}\right) R_{i+1}\left(\theta^{k}\right),
\end{gathered}
$$

$B_{(j)}(\cdot)$ is the $j^{t h}$ column of matrix $B(\cdot), \pi\left(\theta^{k}\right)$ is the probability distribution of $Z_{1}, k \in\{0,1\}$.

Theorem 6. If $\exists C=$ const $>0, \inf _{n} \operatorname{tr}\left(\Gamma \psi(n) \psi^{T}(n)\right) \geq C$, then the characteristics of the test (3),(4) satisfy the following expansions:

$$
\begin{gathered}
E^{(k)}(N)=1+\left(\pi\left(\theta^{k}\right)\right)^{\prime} S\left(\theta^{k}\right) \cdot \mathbf{1}_{K}+O(h), k \in\{0,1\}, \\
\alpha=\left(\pi\left(\theta^{0}\right)\right)^{\prime} B_{(2)}\left(\theta^{0}\right)+\pi_{K+1}\left(\theta^{0}\right)+O(h), \\
\beta=\left(\pi\left(\theta^{1}\right)\right)^{\prime} B_{(1)}\left(\theta^{1}\right)+\pi_{0}\left(\theta^{1}\right)+O(h),
\end{gathered}
$$

where $\left(\pi\left(\theta^{k}\right)\right)^{\prime}=\left(\pi_{1}\left(\theta^{k}\right), \ldots, \pi_{K}\left(\theta^{k}\right)\right)$.

Proof. Under the conditions of Theorem 6 sequence $Z_{n}$ satisfies the conditions (5)-(7) asymptotically as $h \rightarrow 0$. The results of this theorem are derived from the Lemma 4, Theorems 1 and 2 .

\subsection{Robustness evaluation}

In practice the observed data can often come from more complicated sources than hypothetical ones because of the distortion. Some contamination models (see Huber (1981)) can be used 
to analyze the robustness of statistical procedures. However, in this paper we consider only the case where the noise components $\xi_{t}$ in model (1) are distorted by another noises in the Gaussian distribution family.

Suppose that the observed data come from the following mixed model:

$$
\bar{x}_{t}=\theta^{T} \psi(t)+(1-\varepsilon) \xi_{t}+\varepsilon \tilde{\xi}_{t},
$$

where $\left\{\tilde{\xi}_{t}, t \geq 1\right\}$ is a sequence of independent identically distributed random variables, $\tilde{\xi}_{t} \sim$ $N\left(0, \tilde{\sigma}^{2}\right), \tilde{\sigma}$ is a given positive constant, $\xi_{t}$ and $\tilde{\xi}_{t}$ are independent for all $t$, and $\varepsilon \in[0,1 / 2)$ is a level of distortion.

Introduce the notation: $\bar{\lambda}_{t}=\lambda_{t}\left(\bar{x}_{t}\right), \bar{\mu}_{t}^{(k)}=E^{(k)}\left(\bar{\lambda}_{t}\right), \bar{\sigma}_{t}^{2}=D\left(\bar{\lambda}_{t}\right), \bar{m}_{n}^{(k)}=\sum_{i=1}^{n} \bar{\mu}_{i}^{(k)}, \bar{s}_{n}^{2}=$ $\sum_{i=1}^{n} \bar{\sigma}_{i}^{2}$, and $\bar{P}^{(n)}\left(\theta^{k}\right), \bar{R}_{n}\left(\theta^{k}\right), \bar{Q}_{n}\left(\theta^{k}\right), \bar{\pi}\left(\theta^{k}\right), \bar{\alpha}, \bar{\beta}, \bar{t}\left(\theta^{k}\right)$ are calculated analogously by replacing $x_{t}$ with $\bar{x}_{t}$.

For $t, n \geq 1, k \in\{0,1\}$, we get:

$$
\bar{\mu}_{t}^{(k)}=\mu_{t}^{(k)}, \bar{m}_{n}^{(k)}=m_{n}^{(k)}, \bar{\sigma}_{t}^{2}=\frac{\sigma_{t}^{2}}{\sigma^{2}}\left[(1-\varepsilon)^{2} \sigma^{2}+\varepsilon^{2} \tilde{\sigma}^{2}\right], \bar{s}_{n}^{2}=\frac{s_{n}^{2}}{\sigma^{2}}\left[(1-\varepsilon)^{2} \sigma^{2}+\varepsilon^{2} \tilde{\sigma}^{2}\right] .
$$

Theorem 7. For the contaminated model (31), under the conditions of Theorem 6 the following asymptotic expansions hold as $\varepsilon \rightarrow 0$ and $h \rightarrow 0$ :

$$
\begin{gathered}
\bar{\alpha}=\alpha+O(\varepsilon)+O(h), \\
\bar{\beta}=\beta+O(\varepsilon)+O(h), \\
\bar{t}\left(\theta^{k}\right)=t\left(\theta^{k}\right)+O(\varepsilon)+O(h), k \in\{0,1\} .
\end{gathered}
$$

Proof. From (32), as $\varepsilon \rightarrow 0$ we have:

$$
\begin{gathered}
\frac{1}{\bar{\sigma}_{t}^{2}}=\frac{1}{\sigma_{t}^{2}}(1+O(\varepsilon)), \quad \frac{1}{\bar{s}_{n}^{2}}=\frac{1}{s_{n}^{2}}(1+O(\varepsilon)), \\
n_{1}\left(x ; \bar{\mu}_{1}^{(k)}, \bar{\sigma}_{1}^{2}\right)=\frac{1+O(\varepsilon)}{\sigma_{1} \sqrt{2 \pi}} \exp \left[-\frac{\left(x-\mu_{1}^{(k)}\right)^{2}}{2 \sigma_{1}^{2}}(1+O(\varepsilon))\right] \\
=(1+O(\varepsilon)) n_{1}\left(x ; \mu_{1}^{(k)}, \sigma_{1}^{2}\right), \forall x \in \mathbb{R},
\end{gathered}
$$

which implies that

$$
\bar{\pi}_{i}\left(\theta^{k}\right)=\pi_{i}\left(\theta^{k}\right)+O(\varepsilon), i=\overline{0, K+1}, k \in\{0,1\} .
$$

Similarly, we also have as $\varepsilon \rightarrow 0$

$$
n_{1}\left(y ; \bar{m}_{n-1}^{(k)}, \bar{s}_{n-1}^{2}\right) n_{1}\left(x ; y+\bar{\mu}_{n}^{(k)}, \bar{\sigma}_{n}^{2}\right)=(1+O(\varepsilon))^{2} n_{1}\left(y ; m_{n-1}^{(k)}, s_{n-1}^{2}\right) n_{1}\left(x ; y+\mu_{n}^{(k)}, \sigma_{n}^{2}\right),
$$

and obtain

$$
\begin{aligned}
& \bar{r}_{i j}^{(n)}\left(\theta^{k}\right)=r_{i j}^{(n)}\left(\theta^{k}\right)+O(\varepsilon), 1 \leq i \leq K, j=1,2, \\
& \bar{q}_{i j}^{(n)}\left(\theta^{k}\right)=q_{i j}^{(n)}\left(\theta^{k}\right)+O(\varepsilon), 1 \leq i, j \leq K .
\end{aligned}
$$

Combining (36)-(38) and using Theorem 6 we get (33)-(35).

\section{Numerical examples}

First consider the probability model (1) for the special case from Section 4.2 with the following parameters values: $m=4, \sigma=10, \psi(t)=\left(1+1 / t, 4-t / 10, t / 10, t^{2} / 10\right)^{T}, \theta^{0}=$ 
$(1,2,2,1)^{T}, \theta^{1}=(1,1,1,1)^{T}$; hypotheses $(2)$ were tested. Denote the sample estimate of a characteristic $\gamma$ with Monte-Carlo method by $\hat{\gamma}$. The number of simulation runs used in this method was 100 000. Denote $t_{k}=E^{(k)}(N), k=\overline{0,1}$.

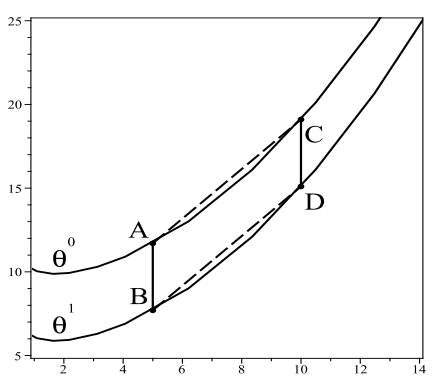

(a)

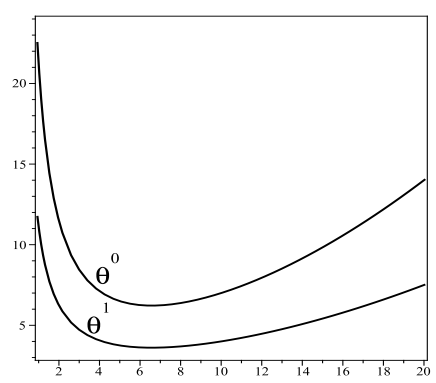

(b)

Figure 1: Trend functions

Suppose that hypothesis $H_{0}$ is true. Then we have $a=\left(\theta^{0}-\theta^{1}\right)^{T} \psi(t)=4, \forall t$. This means that the lengths of segments $\mathrm{AB}$ and $\mathrm{CD}$ are always the same in all positions such that they are parallel to the vertical axis (figure 1a). In addition, $\lambda_{t}, t \geq 1$, are independent identically distributed random variables, $\lambda_{t} \sim N\left(\mu, \sigma_{0}^{2}\right)$, where $\mu=-50, \sigma_{0}=0.4$. MonteCarlo estimates $\left(\hat{\alpha}\right.$ and $\left.\hat{t}_{0}\right)$ and approximate values $\left(\tilde{\alpha}=1-\beta_{\theta^{0}}(0)\right.$ and $\left.\tilde{t}_{0}=\tilde{N}_{\theta^{0}}(0)\right)$ calculated according to Section 4.2 for Type I error probability $\alpha$ and conditional average number of observations $t_{0}$ respectively are presented in Table 1 . When the value of $m_{0}$ increases, the approximate values of test characteristics tend to their corresponding Monte-Carlo estimates.

Table 1: Performance characteristics estimates

\begin{tabular}{|c|c|c|c|c|c|c|}
\hline$\alpha_{0}$ & $\beta_{0}$ & $\hat{\alpha}$ & $\hat{t}_{0}$ & $m_{0}$ & $\tilde{\alpha}$ & $\tilde{t}_{0}$ \\
\hline \multirow{2}{*}{0.1} & \multirow{2}{*}{0.1} & \multirow{2}{*}{0.08034} & \multirow{2}{*}{25.73666} & 200 & 0.08236 & 25.70478 \\
\cline { 5 - 7 } & & & & 500 & 0.08076 & 25.72103 \\
\hline \multirow{2}{*}{0.05} & \multirow{2}{*}{0.1} & \multirow{2}{*}{0.04072} & \multirow{2}{*}{28.46724} & 200 & 0.04128 & 28.46644 \\
\cline { 5 - 7 } & & & 500 & 0.04080 & 28.47073 \\
\hline \multirow{2}{*}{0.05} & \multirow{2}{*}{0.05} & \multirow{2}{*}{0.03990} & \multirow{2}{*}{36.83060} & 200 & 0.03816 & 36.83469 \\
\cline { 5 - 7 } & & & 500 & 0.03974 & 36.80202 \\
\hline
\end{tabular}

The dependence of the operating characteristic and the average sample size functions on the initial value $x$ in the modified test is presented in figure 2 for the case of $m_{0}=200, \alpha_{0}=$ $0.05, \beta_{0}=0.1$. Under hypothesis $H_{0}, \beta_{\theta^{0}}(x)$ is a decreasing function with respect to $x$ (figure $2 \mathrm{a})$. This fact is easily understood because when $x$ increases, the probability that $x+\Lambda_{n}$ comes out of the interval $\left(C_{-}-x, C_{+}-x\right)$ through the upper boundary $C_{+}-x$ also increases. However, function $N_{\theta^{0}}(x)$ increases to the maximum value in the interval $\left(C_{-}, C_{+}\right)$before dropping (figure $2 b$ ). 


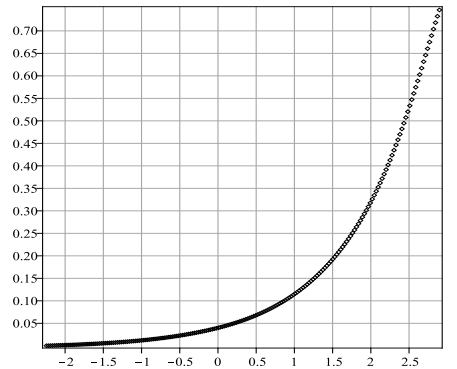

(a)

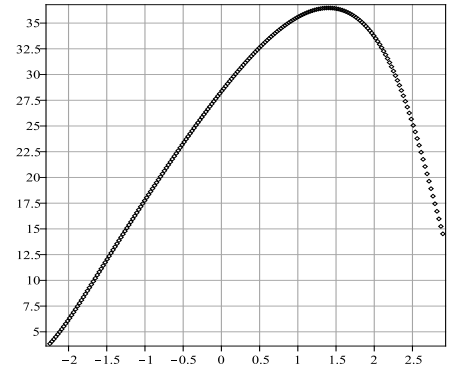

(b)

Figure 2: Plots of functions $1-\beta_{\theta^{0}}(x)$ and $N_{\theta^{0}}(x)$

In the next examples, the following values of parameters are used for calculating: $m=$ $4, \sigma=10, \psi(t)=\left(1, t / 10, t^{2} / 100,10 / t\right)^{T}, \theta^{0}=(1,2,2,2)^{T}, \theta^{1}=(1,1,1,1)^{T}$. In this case, $P_{k}\left(\Lambda_{50} \in\left(C_{-}, C_{+}\right)\right) \leq 10^{-6}, k \in\{0,1\}$. All infinite sums were replaced by the sums of the first 50 summands, this provides the accuracy of the order 0.00001 . Figure $1 \mathrm{~b}$ shows the plots of trend functions. The upper bounds for the test performance characteristics constructed in Corollary 3 are given in Tables 2 and 3 .

Table 2: The upper bounds for error probabilities

\begin{tabular}{|c|c|c|c|c|c|}
\hline$\alpha_{0}$ & $\beta_{0}$ & $\hat{\alpha}$ & $\alpha \leq$ & $\hat{\beta}$ & $\beta \leq$ \\
\hline 0.1 & 0.1 & 0.07166 & 0.12155 & 0.07244 & 0.12155 \\
\hline 0.05 & 0.1 & 0.03392 & 0.05307 & 0.07006 & 0.11402 \\
\hline 0.01 & 0.05 & 0.00534 & 0.00761 & 0.03216 & 0.04734 \\
\hline
\end{tabular}

Table 3: The upper bounds for the average number of observations

\begin{tabular}{|c|c|c|c|c|c|}
\hline$\alpha_{0}$ & $\beta_{0}$ & $\hat{t}_{0}$ & $E^{(0)}(N) \leq$ & $\hat{t}_{1}$ & $E^{(1)}(N) \leq$ \\
\hline 0.1 & 0.1 & 16.07760 & 18.09357 & 16.03320 & 18.09357 \\
\hline 0.05 & 0.1 & 17.03316 & 18.68805 & 19.70408 & 21.30107 \\
\hline 0.01 & 0.05 & 21.13740 & 22.13213 & 25.61644 & 26.41215 \\
\hline
\end{tabular}

Denote the main terms of asymptotic expansions of $\alpha, \beta, E^{(k)}(N)$ respectively by $\bar{\alpha}_{A S Y M}$, $\bar{\beta}_{A S Y M}, \bar{E}_{A S Y M}^{(k)}(N)$. The numerical results for these main terms are presented in Table 4 .

Table 4: The main terms of asymptotic expansions of the test characteristics

\begin{tabular}{|c|c|c|c|c|c|c|}
\hline$\alpha_{0}$ & $\beta_{0}$ & $K$ & $\bar{\alpha}_{A S Y M}$ & $\bar{\beta}_{A S Y M}$ & $\bar{E}_{A S Y M}^{(0)}(N)$ & $\bar{E}_{A S Y M}^{(1)}(N)$ \\
\hline \multirow{3}{*}{0.1} & \multirow{3}{*}{0.1} & 10 & 0.07449 & 0.07449 & 15.85942 & 15.85942 \\
\cline { 3 - 7 } & 20 & 0.07273 & 0.07273 & 16.03470 & 16.03470 \\
\cline { 3 - 7 } 0.05 & \multirow{3}{*}{0.1} & 40 & 0.07225 & 0.07225 & 16.08029 & 16.08029 \\
\hline & & 20 & 0.03501 & 0.07367 & 16.76240 & 19.49727 \\
\hline & & 40 & 0.03433 & 0.07104 & 16.97260 & 19.65483 \\
\hline \multirow{3}{*}{0.01} & \multirow{2}{*}{0.05} & 10 & 0.00586 & 0.07033 & 17.02777 & 19.69613 \\
\cline { 3 - 7 } & & 20 & 0.00579 & 0.03291 & 21.06344 & 25.65018 \\
\cline { 3 - 7 } & 40 & 0.00576 & 0.0324 & 21.12768 & 25.67067 \\
\hline
\end{tabular}




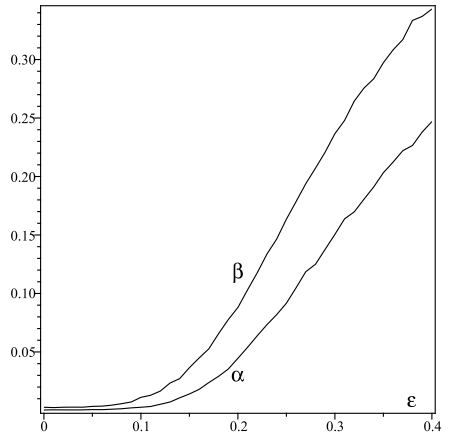

(a)

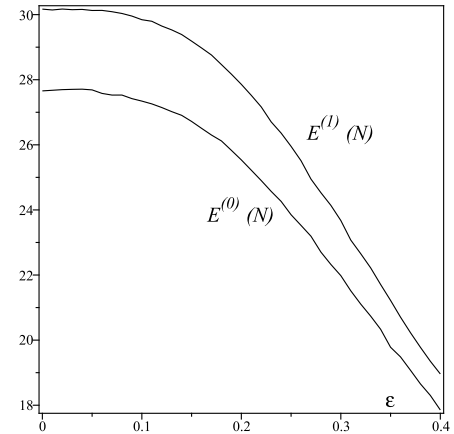

(b)

Figure 3: Dependence of performance characteristics on probability of contamination $\varepsilon$

Due to symmetric property, we have $\bar{\alpha}_{A S Y M}=\bar{\beta}_{A S Y M}, \bar{E}_{A S Y M}^{(0)}(N)=\bar{E}_{A S Y M}^{(1)}(N)$ provided $\alpha_{0}=\beta_{0}$. When the value $K$ increases, the main terms of asymptotic expansions of the test characteristics become closer to their corresponding Monte-Carlo estimates (see Tables 2 and $3)$. The orders of approximation in (28)-(30) are only $O(h)$. Therefore, if we want to make the main terms of asymptotic expansions better, the value $K$ must be larger. However, with the large value $K$ the computation on infinite sums (in practice, they can be reasonably replaced with finite ones because of the termination of the test) of matrices with high dimensions $S\left(\theta^{i}\right)$ and $B\left(\theta^{i}\right)$ will cost much time.

Figure 3 shows the dependence of the error probabilities and average number of observations on the probability of contamination $\varepsilon$ in the model (31), when $\tilde{\sigma}^{2}=50 \sigma^{2}, \alpha_{0}=0.001, \beta_{0}=$ 0.005 . When the contamination probability $\varepsilon$ increases, both error probabilities increase. For both conditional average numbers of observations, there are opposite pictures.

\section{Conclusion}

The problem of sequential testing for time series with trend is studied. The sufficient condition of termination of the test is given. Beside the explicit (but not useful for further analysis) formulae for the test characteristics, an approach to approximate test characteristics is also constructed. This approach allows us not only to estimate the test characteristics, but also to analyze the robustness of the test.

\section{References}

Basseville M, Nikiforov I (1993). Detection of Abrupt Changes: Theory and Application. Prentice Hall.

Bilodeau M, Brenner D (1999). The Theory of Multivariate Statistics. Springer-Verlag.

Coope I (1996). "On Matrix Trace Inequalities and Related Topics for Products of Hermitian Matrices." Journal of Mathematical Analysis and Applications, 188, 999-1001.

Cox D, Miller H (1965). The Theory of Stochastic Processes. John Wiley and Sons.

Govindarajulu Z (2004). Sequential Statistics. World Scientifc.

Gut A (2005). Probability: A Graduate Course. Springer Science-Business Media Inc.

Hoffman J (2001). Numerical Methods for Engineers and Scientists. Marcel Dekker Inc.

Huber P (1981). Robust Statistics. John Wiley and Sons. 
Kharin A (2005). "Robust Bayesian Prediction under Distortions of Prior and Conditional Distributions." Journal of Mathematical Sciences, 126(1), 992-997.

Kharin A (2008). "Robustness Evaluation in Sequential Testing of Composite Hypotheses." Austrian Journal of Statistics, 37(1), 51-60.

Kharin A (2011). "Robustness Analysis for Bayesian Sequential Testing of Composite Hypotheses under Simultaneous Distortion of Priors and Likelihoods." Austrian Journal of Statistics, 40(1\&2), 65-73.

Kharin A (2013). "Robustness of Sequential Testing of Hypotheses on Parameters of M-valued Random Sequences." Journal of Mathematical Sciences, 189(6), 924-931.

Kharin A (2016). "Performance and Robustness Evaluation in Sequential Hypotheses Testing." Communications in Statistics - Theory and Methods, 45(6), 1693-1709.

Kharin A, Kishylau D (2015). "Robust Sequential Test for Hypotheses about Discrete Distributions in the Presence of "Outliers"." Journal of Mathematical Sciences, 205(1), 68-73.

Kharin A, Ton T (2016). "Sequential Statistical Hypotheses Testing on Parameters of Time Series with a Trend under Missing Values." Proceedings of the National Academy of Sciences of Belarus. Series of Physical-Mathematical Sciences, (3), 38-46.

Kharin Y (1997). "Robustness of Clustering under Outliers." Lecture Notes in Computer Science, 1280, 501-512.

Maevskii V, Kharin Y (2002). "Robust Regressive Forecasting under Functional Distortions in a Model." Automation and Remote Control, 63(11), 1803-1820.

Wald A (1947). Sequential Analysis. John Wiley and Sons.

Wald A, Wolfowitz J (1948). "Optimum Character of the Sequential Probability Ratio Test." The Annals of Mathematical Statistics, 19(3), 326-339.

\section{Affiliation:}

Alexey Kharin, Ton That Tu

Department of Probability Theory and Mathematical Statistics

Faculty of Applied Mathematics and Informatics

Belarusian State University

Independence avenue, 4

Minsk 220030

Belarus

E-mail: KharinAY@bsu.by

Ton That $\mathrm{Tu}$

Faculty of Mathematics

Da Nang University of Education

Ton Duc Thang street, 459

Da Nang 555726

Vietnam

tthattu@gmail.com

\section{Austrian Journal of Statistics}

published by the Austrian Society of Statistics

Volume 46

April 2017 http://www.ajs.or.at/

http://www.osg.or.at/

Submitted: 2016-11-15

Accepted: 2017-02-02 